\title{
Investigation of hidden-charm pentaquarks with strangeness $S=-1$
}

\author{
Xiaohuang Hu, Jialun Ping ${ }^{\mathrm{a}}$ \\ Department of Physics and Jiangsu Key Laboratory for Numerical Simulation of Large Scale Complex Systems, Nanjing Normal University, \\ Nanjing 210023, People's Republic of China
}

Received: 24 October 2021 / Accepted: 21 January 2022 / Published online: 7 February 2022

(C) The Author(s) 2022

\begin{abstract}
Recently, a new hidden-charm pentaquark state $P_{c s}(4459)$ was reported by the LHCb Collaboration. Stimulated by the fact that all hidden-charm pentaquark states in $S=0$ systems were successfully studied by the chiral quark model, we extended this study to the $S=-1$ systems. All possible states with quantum numbers $I J^{P}=0\left(\frac{1}{2}\right)^{-}$, $0\left(\frac{3}{2}\right)^{-}, 0\left(\frac{5}{2}\right)^{-}, 1\left(\frac{1}{2}\right)^{-}, 1\left(\frac{3}{2}\right)^{-}$and $1\left(\frac{5}{2}\right)^{-}$have been investigated. The calculation results shows that the newly observed state $P_{c s}(4459)$ can be explained as $\Xi_{c} \bar{D}^{*}$ molecular state and the quantum numbers are $0\left(\frac{1}{2}\right)^{-}$. In addition, we also find other molecular states $\Xi_{c} \bar{D}, \Xi_{c}^{*} \bar{D}$ and $\Xi_{c}^{\prime} \bar{D}^{*}$. It is worth mentioning that $\Xi_{c} \bar{D}^{*}$ can form a two-peak structure from states in system $0\left(\frac{1}{2}\right)^{-}$and $0\left(\frac{3}{2}\right)^{-}$. The decay width of all molecular states is given with the help of real scaling method. These hidden-charm pentaquark states is expected to be further verified in future experiments.
\end{abstract}

\section{Introduction}

Since $\Theta^{+}$was proposed in $2003[1,2]$, the search of pentaquark states has always been a hot topic. A large number of exotic hadronic states, " $X Y Z$ " states which associated with tetraquark, have been discovered in experiments over the past decade. Naturally the observation of pentaquark state is expected. The important and prominent event arrived in 2015, the $P_{c}^{+}$states $P_{c}^{+}(4380)$ and $P_{c}^{+}(4450)$, which are the strongest candidates for pentaquark states were observed by the LHCb Collaboration in the $J / \psi p$ invariant mass spectrum of decay channel $\Lambda_{b} \rightarrow J \psi p K$ [3]. And then in 2019, the LHCb Collaboration reanalysed the same process with more data and updated their results, which shows that $P_{c}^{+}(4450)$ can split into two structures, $P_{c}^{+}(4440)$ and $P_{c}^{+}$(4457), while $P_{c}^{+}$(4312) is identified as a new structure [4]. These $P_{c}^{+}$states all have a common feature that

a e-mail: jlping@njnu.edu.cn (corresponding author) they are very close to baryon-meson thresholds, which led to one of the most popular interpretation of these $P_{c}^{+}$states as molecular states [5-11]. Other possible explanations also exist, such as the compact pentaquark states $[12,13]$. In addition, the decay properties and width of these $P_{c}^{+}$states also have been investigated in Ref. [14].

Very recently, the LHCb Collaboration reported a new pentaquark state with strangeness, $P_{c s}^{+}(4459)$ in the $J / \psi \Lambda$ invariant mass spectrum of decay channel $\Xi_{b} \rightarrow J \psi K^{-} \Lambda$ [15]. The mass and decay width of $P_{c s}^{+}(4459)$ are,

$$
\begin{aligned}
& M=4458.8 \pm 2.9_{-1.1}^{+4.7} \mathrm{MeV}, \\
& \Gamma=17.3 \pm 6.0_{-5.7}^{+8.0} \mathrm{MeV},
\end{aligned}
$$

while its spin and parity are unknown. Like other $P_{c}^{+}$pentaquark states, the mass of $P_{c S}^{+}(4459)$ is very close to threshold of $\Xi_{c} \bar{D}^{*}$. Thus, some phenomenological models have been used to investigate whether $P_{c S}^{+}$(4459) can be explained as a $\Xi_{c} \bar{D}^{*}$ molecular state, such as QCD sum rule $[16,17]$, coupled channel unitary approach [18], effective field theory [19] and so on [20-23]. Other structures of $P_{c s}$ are also proposed, for example, diquark-diquark-antiquark structure [24]. Actually, there are many theoretical work on $P_{c s}$ state before the experiment [25-28].

It is important to highlight here that, before the LHCb's discovered several $P_{c}^{+}$pentaquark states, their existence was predicted [29-31], and our quark model calculation also gave a good description and prediction [32]. $P_{c}^{+}(4312), P_{c}^{+}$(4440) and $P_{c}^{+}(4459)$ were described as baryon-meson molecular states with the $I J^{P}=\frac{1}{2}\left(\frac{1}{2}\right)^{-} \Sigma_{c} \bar{D}, \frac{1}{2}\left(\frac{1}{2}\right)^{-} \Sigma_{c} \bar{D}^{*}$ and $\frac{1}{2}\left(\frac{3}{2}\right)^{-} \Sigma_{c} \bar{D}^{*}$, respectively. A lot of subsequent theoretical work also supports our conclusion [9-11]. Thus, it is natural to extend herein such study from $P_{c}$ states to the $P_{c s}$ states. Since the quark model was proposed by Gell-Mann and Zweig in 1964 respectively [33,34], it has become the most common approach to study the multiquark system as it evolves. In this work, the constituent chiral quark model 
(ChQM) will still be employed to investigate $q q s c \bar{c}$ systems ( $q$ stands for $u$ or $d$ ) corresponding to $P_{c s}$ states. To calculate accurately the results of each possible system, Gaussian expansion method (GEM) [35], an accurate and universal few-body calculation method is adopted. The GEM is very suitable for the calculation of few-body systems. Within this method, the orbital wave functions of all relative motions of the systems are expanded by gaussians. After considering all possible configuration of color, spin and flavor degrees of freedom, we can identify the structures of the system. Finally, with the help of "real scaling method", we can confirm the genuine five-quark resonances and their respective decay widths along.

The paper is organized as follow. After introduction, details of ChQM and GEM are introduced in Sect. 2. In Sect. 3, we present the method of finding and calculating the decay width of the genuine resonance state ("real scaling method"), and then we show the results with analysis and discussion of $P_{c s}$ structure. Finally, We give a brief summary of this work in the last section.

\section{Chiral quark model and wave functions}

In this paper, ChQM is employed to investigate the $P_{c s}$ states. The model has become one of the most common approaches to describe hadron spectra, hadron-hadron interactions and multiquark states [36]. In this model, in addition to one-gluon exchange (OGE), the massive constituent quarks also interact with each other through Goldstone boson exchange. Besides, the color confinement and the scalar $\sigma$ meson (chiral partner, acting on $u$ and $d$ quark only) exchange are also introduced. More details of this model can be found in Refs. [36,37]. The Hamiltonian of ChQM is given as follows:

$$
\begin{aligned}
H & =\sum_{i=1}^{n}\left(m_{i}+\frac{p_{i}^{2}}{2 m_{i}}\right)-T_{C M}+\sum_{j>i=1}^{n} V_{i j} \\
V_{i j} & =V_{i j}^{C}+V_{i j}^{G}+V_{i j}^{\chi}+V_{i j}^{\sigma}, \\
V_{i j}^{C}= & \lambda_{i}^{c} \cdot \lambda_{j}^{c}\left[-a_{c}\left(1-e^{-\mu_{c} r_{i j}}\right)+\Delta\right], \\
V_{i j}^{G}= & \frac{\alpha_{s}}{4} \lambda_{i}^{c} \cdot \lambda_{j}^{c}\left[\frac{1}{r_{i j}}-\frac{1}{6 m_{i} m_{j}} \sigma_{i} \cdot \sigma_{j} \frac{e^{-r_{i j} / r_{0}(\mu)}}{r_{i j} r_{0}^{2}(\mu)}\right], \\
r_{0}(\mu)= & \hat{r}_{0} / \mu, \quad \alpha_{s}=\frac{\alpha_{0}}{\ln \left(\frac{\mu^{2}+\mu_{0}^{2}}{\Lambda_{0}^{2}}\right)} . \\
V_{i j}^{\chi}= & \left.v_{\pi}\left(\mathbf{r}_{i j}\right) \sum_{a=1}^{3} \lambda_{i}^{a} \lambda_{j}^{a}+v_{K}\left(\mathbf{r}_{i j}\right) \sum_{a=4}^{7} \lambda_{i}^{a} \lambda_{j}^{a}\right) \\
& +v_{\eta}\left(\mathbf{r}_{i j}\right)\left[\cos \theta_{P}\left(\lambda_{i}^{8} \lambda_{j}^{8}\right)-\sin \theta_{P}\right], \\
v_{i j}^{\chi}= & \frac{g_{c h}^{2}}{4 \pi} \frac{m_{\chi}^{2}}{12 m_{i} m_{j}} \frac{\Lambda_{\chi}^{2}}{\Lambda_{\chi}^{2}-m_{\chi}^{2}} m_{\chi}
\end{aligned}
$$

Table 1 Quark model parameters

\begin{tabular}{lll}
\hline Quark masses & $m_{u}=m_{d}(\mathrm{MeV})$ & 313 \\
& $m_{s}(\mathrm{MeV})$ & 555 \\
& $m_{c}(\mathrm{MeV})$ & 1780 \\
Goldstone bosons & $\Lambda_{\pi}=\Lambda_{\sigma}\left(\mathrm{fm}^{-1}\right)$ & 4.20 \\
& $\Lambda_{\eta}=\Lambda_{K}\left(\mathrm{fm}^{-1}\right)$ & 5.20 \\
& $m_{\pi}\left(\mathrm{fm}^{-1}\right)$ & 0.70 \\
& $m_{K}\left(\mathrm{fm}^{-1}\right)$ & 2.51 \\
& $m_{\eta}\left(\mathrm{fm}^{-1}\right)$ & 2.77 \\
& $m_{\sigma}\left(\mathrm{fm}^{-1}\right)$ & 3.42 \\
& $g_{c h}^{2} /(4 \pi)$ & 0.54 \\
& $\theta_{P}\left({ }^{\circ}\right)$ & -15 \\
& $a_{c}(\mathrm{MeV})$ & 280.3 \\
Confinement & $\left.\mu_{c}(\mathrm{fm})^{-1}\right)$ & 0.863 \\
& $\Delta(\mathrm{MeV})$ & 115.0 \\
& $\hat{r}_{0}(\mathrm{MeVfm})$ & 49.3 \\
& $\alpha_{u u}$ & $0.623 / 0.924$ \\
& $\alpha_{u s}$ & $0.915 /-$ \\
& $\alpha_{u c}$ & $0.900 / 0.765$ \\
& $\alpha_{s c}$ & $0.710 / 0.633$ \\
& $\alpha_{c c}$ & $-/ 0.5$ \\
\hline
\end{tabular}

$$
\begin{aligned}
& \times\left[Y\left(m_{\chi} r_{i j}\right)-\frac{\Lambda_{\chi}^{3}}{m_{\chi}^{3}} Y\left(\Lambda_{\chi} r_{i j}\right)\right]\left(\sigma_{i} \cdot \sigma_{j}\right), \\
\chi= & \pi, K, \eta \\
V_{i j}^{\sigma}= & -\frac{g_{c h}^{2}}{4 \pi} \frac{\Lambda_{\sigma}^{2} m_{\sigma}}{\Lambda_{\sigma}^{2}-m_{\sigma}^{2}}\left[Y\left(m_{\sigma} r_{i j}\right)-\frac{\Lambda_{\sigma}}{m_{\sigma}} Y\left(\Lambda_{\sigma} r_{i j}\right)\right] .
\end{aligned}
$$

where $T_{c m}$ is the kinetic energy of the center-of mass motion and $\mu$ is the reduced mass between two interacting quarks. Only the central parts of the interactions are given here because we are interested in the low-lying states of the multiquark system. $\sigma$ represents the SU(2) Pauli matrices; $\lambda^{c}$ and $\lambda$ represent the SU(3) color and flavor Gell-Mann matrices respectively; $\alpha_{S}$ denotes the strong coupling constant of onegluon exchange and $Y(x)$ is the standard Yukawa functions. Because it is difficult to use the same set of parameters to have a good description of baryon and meson spectra simultaneously, we treat the strong coupling constant of one-gluon exchange with different values for quark-quark and quarkantiquark interacting pairs.

The model parameters are listed in Table 1, and the calculated baryon and meson masses are presented in the Table 2 with the experimental values. From the calculation,most of the results are close to experimental values except for the $\Lambda_{c}$ and $J / \psi$. In the follow-up calculation, we find that the molecular state corresponding to these two hadrons are open channels and these calculation errors do not cause mass inversion, so these errors do not affect our final results. 
Table 2 The masses of ground-state baryons and mesons (unit: Mev)

\begin{tabular}{lcccccc}
\hline & $\Lambda$ & $\Sigma$ & $\Sigma^{*}$ & $\Lambda_{c}$ & $\Sigma_{c}^{\prime}$ & $\Sigma_{c}^{*}$ \\
\hline CHQM & 1114 & 1243 & 1404 & 2184 & 2453 & 2529 \\
Expt & 1116 & 1189 & 1385 & 2286 & 2455 & 2520 \\
\hline & $\Xi_{c}$ & \multicolumn{2}{c}{$\Xi_{c}^{\prime}$} & $\Xi_{c}^{*}$ & & \\
\hline CHQM & 2460 & 2580 & 2653 & & \\
Expt & 2471 & 2589 & 2645 & & \\
\hline & $\pi$ & $\rho$ & $D$ & $D^{*}$ & $D_{s}$ & $D_{s}^{*}$ \\
\hline CHQM & 140 & 698 & 1858 & 2023 & 1964 & 2156 \\
Expt & 140 & 775 & 1864 & 2007 & 1968 & 2112 \\
\hline \multicolumn{7}{c}{$\eta_{c}$} \\
\hline CHQM & 2984 & 3182 & & & \\
Expt & 2984 & 3097 & & & \\
\hline
\end{tabular}

In the following, the wave functions for the five-quark systems are constructed and the eigen-energy is obtained by solving the Schrödinger equation. The wave function of the system consists of four parts: orbital, spin, flavor and color. The wave function of each part is constructed in two steps, first construct the wave function of three-quark cluster and quark-antiquark cluster, respectively, then coupling two clusters wave functions to form the complete five-body one. In the following, the wave functions for $(q q s)(\bar{c} c)$ configuration is written down, the wave functions for other configuration can be obtained by exchange the indices of particles. The indices of particles $q, q, s, \bar{c}, c$ are $1,2,3,4,5$. The wave functions for $(q q c)(\bar{c} s)$ are obtained by exchange the particle indices $3 \leftrightarrow 5$ as an example.

The first part is orbital wave function. A five-body system have four relative motions so it is written as follows.

$$
\psi_{L M_{L}}^{x}=\left[\left[\left[\psi_{n_{1} l_{1}}(\boldsymbol{\rho}) \psi_{n_{2} l_{2}}(\boldsymbol{\lambda})\right]_{l} \psi_{n_{3} l_{3}}(\boldsymbol{r})\right]_{l^{\prime}} \psi_{n_{4} l_{4}}(\boldsymbol{R})\right]_{L M_{L}},
$$

where the Jacobi coordinates are defined as follows,

$$
\begin{aligned}
\boldsymbol{\rho} & =\boldsymbol{x}_{1}-\boldsymbol{x}_{2}, \\
\lambda & =\left(\frac{m_{1} \boldsymbol{x}_{1}+m_{2} \boldsymbol{x}_{2}}{m_{1}+m_{2}}\right)-\boldsymbol{x}_{3}, \\
\boldsymbol{r} & =\boldsymbol{x}_{4}-\boldsymbol{x}_{5} \\
\boldsymbol{R} & =\left(\frac{m_{1} \boldsymbol{x}_{1}+m_{2} \boldsymbol{x}_{2}+m_{3} \boldsymbol{x}_{3}}{m_{1}+m_{2}+m_{3}}\right)-\left(\frac{m_{4} \boldsymbol{x}_{4}+m_{5} \boldsymbol{x}_{5}}{m_{4}+m_{5}}\right) .
\end{aligned}
$$

$\boldsymbol{x}_{i}$ is the position of the $i$-th particle. Then we use a set of gaussians to expand the radial part of the orbital wave func- tion which is shown below,

$$
\begin{aligned}
& \psi_{l m}(\mathbf{r})=\sum_{n=1}^{n_{\max }} c_{n l} \phi_{n l m}^{G}(\mathbf{r}) \\
& \phi_{n l m}^{G}(\mathbf{r})=N_{n l} r^{l} e^{-v_{n} r^{2}} Y_{l m}(\hat{\mathbf{r}})
\end{aligned}
$$

where $N_{n l}$ is the normalization constant,

$N_{n l}=\left(\frac{2^{l+2}\left(2 v_{n}\right)^{l+3 / 2}}{\sqrt{\pi}(2 l+1) ! !}\right)^{\frac{1}{2}}$,

and $c_{n l}$ is the variational parameter, which is determined by the dynamics of the system. The Gaussian size parameters are chosen according to the following geometric progression:

$v_{n}=\frac{1}{r_{n}^{2}}, r_{n}=r_{\min } a^{n-1}, a=\left(\frac{r_{\max }}{r_{\min }}\right)^{\frac{1}{n_{\max }-1}}$,

where $n_{\max }$ is the number of Gaussian functions, and $n_{\max }$ is determined by the convergence of the results. In the present calculation, $n_{\max }=8$.

The details of constructing flavor, color and spin wave functions of 5-quark system can be found in Ref. [38], only the last expressions are shown here.

Flavor wave functions:

$\left|\chi_{0,0}^{f 1}\right\rangle=\frac{1}{\sqrt{2}}(u d s \bar{c} c-d u s \bar{c} c)$

$\left|\chi_{0,0}^{f 2}\right\rangle=u u s \bar{c} c$

Color wave functions:

$$
\begin{aligned}
\left|\chi^{c 1}\right\rangle= & \frac{1}{\sqrt{18}}(r g b-r b g+g b r-g r b+b r g-b g r) \\
& (\bar{r} r+\bar{g} g+\bar{b} b) \\
\left|\chi^{c 2}\right\rangle= & \frac{1}{\sqrt{192}}[2(2 r r g-r g r-g r r) \bar{r} b \\
& +2(r g g+g r g-2 g g r) \bar{g} b \\
& -2(2 r r b-r b r-b r r) \bar{r} g-2(r b b+b r b-2 b b r) \bar{b} g \\
& +2(2 g g b-g b g-b g g) \bar{g} r+2(g b b+b g b-2 b b g) \bar{b} r \\
& +(r b g-g b r+b r g-b g r)(2 \bar{b} b-\bar{r} r-\bar{g} g) \\
& +(2 r g b-r b g+2 g r b-g b r-b r g-b g r)(\bar{r} r-\bar{g} g)] \\
\left|\chi^{c 3}\right\rangle= & \frac{1}{24}[6(r g r-g r r) \bar{r} b+6(r g g-g r g) \bar{g} b \\
& -6(r b r-b r r) \bar{r} g-6(r b b-b r b) \bar{b} g \\
& +6(g b g-b g g) \bar{g} r+6(g b b-b g b) \bar{b} r \\
& +3(r b g+g b r-b r g-b g r)(\bar{r} r-\bar{g} g) \\
& +(2 r g b+r b g-2 g r b-g b r-b r g+b g r) \\
& \times(2 \bar{b} b-\bar{r} r-\bar{g} g)]
\end{aligned}
$$

Spin wave functions:

$$
\begin{aligned}
\left|\chi_{\frac{1}{2}, \frac{1}{2}}^{\sigma 1}\right\rangle= & \frac{1}{\sqrt{12}}(2 \alpha \alpha \beta \alpha \beta-2 \alpha \alpha \beta \beta \alpha+\alpha \beta \alpha \beta \alpha \\
& -\alpha \beta \alpha \alpha \beta+\beta \alpha \alpha \beta \alpha-\beta \alpha \alpha \alpha \beta)
\end{aligned}
$$




$$
\begin{aligned}
\left|\chi_{\frac{1}{2}, \frac{1}{2}}^{\sigma 2}\right\rangle= & \frac{1}{2}(\alpha \beta \alpha \alpha \beta-\alpha \beta \alpha \beta \alpha+\beta \alpha \alpha \beta \alpha-\beta \alpha \alpha \alpha \beta) \\
\left|\chi_{\frac{1}{2}, \frac{1}{2}}^{\sigma 3}\right\rangle= & \frac{1}{6}(2 \alpha \alpha \beta \alpha \beta+2 \alpha \alpha \beta \beta \alpha-\alpha \beta \alpha \alpha \beta-\alpha \beta \alpha \beta \alpha \\
& -\beta \alpha \alpha \beta \alpha-\beta \alpha \alpha \alpha \beta-2 \alpha \beta \beta \alpha \alpha-2 \beta \alpha \beta \alpha \alpha \\
& +4 \beta \beta \alpha \alpha \alpha) \\
\left|\chi_{\frac{1}{2}, \frac{1}{2}}^{\sigma 4}\right\rangle= & \frac{1}{\sqrt{12}}(\alpha \beta \alpha \alpha \beta+\alpha \beta \alpha \beta \alpha-\beta \alpha \alpha \alpha \beta-\beta \alpha \alpha \beta \alpha \\
& +2 \beta \alpha \beta \alpha \alpha-2 \alpha \beta \beta \alpha \alpha) \\
\left|\chi_{\frac{1}{2}, \frac{1}{2}}^{\sigma 5}\right\rangle= & \frac{1}{\sqrt{18}}(3 \alpha \alpha \alpha \beta \beta-\alpha \alpha \beta \alpha \beta-\alpha \alpha \beta \beta \alpha \\
& -\alpha \beta \alpha \alpha \beta-\alpha \beta \alpha \beta \alpha-\beta \alpha \alpha \alpha \beta-\beta \alpha \alpha \beta \alpha \\
& +\beta \beta \alpha \alpha \alpha+\beta \alpha \beta \alpha \alpha+\alpha \beta \beta \alpha \alpha) \\
\left|\chi_{\frac{3}{2}, \frac{3}{2}}^{\sigma 1}\right\rangle= & \frac{1}{\sqrt{6}}(2 \alpha \alpha \beta \alpha \alpha-\alpha \beta \alpha \alpha \alpha-\beta \alpha \alpha \alpha \alpha) \\
\left|\chi_{\frac{3}{2}, \frac{3}{2}}^{\sigma 2}\right\rangle= & \frac{1}{\sqrt{2}}(\alpha \beta \alpha \alpha \alpha-\beta \alpha \alpha \alpha \alpha) \\
\left|\chi_{\frac{3}{2}, \frac{3}{2}}^{\sigma 3}\right\rangle= & \frac{1}{\sqrt{2}}(\alpha \alpha \alpha \alpha \beta-\alpha \alpha \alpha \beta \alpha) \\
\left|\chi_{\frac{3}{2}, \frac{3}{2}}^{\sigma 4}\right\rangle= & \frac{1}{\sqrt{30}}(2 \alpha \alpha \beta \alpha \alpha+2 \alpha \beta \alpha \alpha \alpha+2 \beta \alpha \alpha \alpha \alpha \\
\left|\chi_{\frac{5}{2}, \frac{5}{2}}^{\sigma 1}\right\rangle= & -3 \alpha \alpha \alpha \alpha \alpha \beta-3 \alpha \alpha \alpha \beta \alpha) \\
& \alpha \alpha \alpha \beta
\end{aligned}
$$

where $\chi^{c 1}$ represents the color wave function of a color singlet-singlet structure, $\chi^{c 2}$ and $\chi^{c 3}$ represent the color octet-octet wave functions respectively. The subscripts of $\chi_{I, I_{z}}^{f}\left(\chi_{S, S_{z}}^{\sigma}\right)$ are total isospin (spin) and its third projection.

Finally, the total wave function of the five-quark system is written as:

$\Psi_{J M_{J}}^{i, j, k}=\mathcal{A}\left[\left[\psi_{L} \chi_{S}^{\sigma_{i}}\right]_{J M_{J}} \chi_{j}^{f i} \chi_{k}^{c i}\right]$

where the $\mathcal{A}$ is the antisymmetry operator of the system which guarantees the antisymmetry of the total wave functions when identical particles exchange. Under our numbering scheme, the antisymmetry operator is

$\mathcal{A}=1-(12)$

At last, we solve the following Schrödinger equation to obtain eigen-energies of the system,

$$
H \Psi_{J M_{J}}=E \Psi_{J M_{J}}
$$

with the help of the Rayleigh-Ritz variational principle. The matrix elements of Hamiltonian can be easily obtained if all the orbital angular momenta are zero, which is reasonable for only considering the low-lying states of five-quark system. It is worthwhile to mention that if the orbital angular momenta of the system are not zero, it is necessary to use the infinitesimally shifted Gaussian method to calculate the matrix elements [35].

\section{Results and discussions}

In this section, we present the calculation results of all lowlying states of the $(u u c)(s \bar{c}),(u u s)(c \bar{c})$ and $(u s c)(u \bar{c})$ fivequark system with all possible quantum numbers $I J^{P}=$ $0\left(\frac{1}{2}\right)^{-}, 0\left(\frac{3}{2}\right)^{-}, 0\left(\frac{5}{2}\right)^{-}, 1\left(\frac{1}{2}\right)^{-}, 1\left(\frac{3}{2}\right)^{-}$and $1\left(\frac{5}{2}\right)^{-}$in ChQM. All the orbital angular momentum of the system is treated as zero and the corresponding parity is negative.

In Tables 3, 4 and 5, the important calculation results are shown. In each table, columns 2 to 5 represent flavor, spin and color each wave functions in each channel and the corresponding physical channel of five-quark system. In column 6 , the eigen-energy of the each channel is listed and the theoretical threshold (the sum of the theoretical masses of corresponding baryon and meson) is given in column 7 . Column 8 gives the binding energies, which are the difference between the eigen-energy and the theoretical threshold. Finally, the experimental thresholds (the sum of the experimental masses of the corresponding baryon and meson) along with corrected energies (the sum of experimental threshold and the binding energy, $E^{\prime}=E_{B}+E_{t h}^{\text {exp }}$ ) are given in last two columns. With this correction, the calculation error caused by the model parameters in five-quark calculation can be reduced partly.

Since we hardly found any bound state in the quantum number of $I=1$ systems, we do not present the results of these systems but focused our analysis on systems with $I J^{P}=0 \frac{1}{2}^{-}, I J^{P}=0 \frac{3}{2}^{-}$and $I J^{P}=0 \frac{5}{2}^{-}$. The results are analyzed in the following:

(a) For $I J^{P}=0 \frac{1}{2}^{-}$system in Table 3: First, the single channel calculations show that there exist weakly bound states in the $\Xi_{c} \bar{D}, \Xi_{c} \bar{D}^{*}$ and $\Xi_{c}^{\prime} \bar{D}$ channels. After coupling to respective hidden-color channels, the attractions all increase by a few MeVs, which is the typical range of binding energy of hadronic molecules. The coupling of all color singlet-singlet channels does not push the lowest energy of $\Lambda \eta_{c}$ below its threshold. Then, a full-channel coupling is made and the results show no bound state can be formed. However, resonances are possible because the attractions exist in the $\Xi_{c} \bar{D}, \Xi_{c} \bar{D}^{*}$ and $\Xi_{c}^{\prime} \bar{D}$ channels. Thus, real-scaling method is required to identify resonances.

(b) For $I J^{P}=0 \frac{3}{2}^{-}$system in Table 4: The single channel calculation results show there are weakly bound states in three single channels, $\Xi_{c} \bar{D}^{*}, \Xi_{c}^{\prime} \bar{D}^{*}$ and $\Xi_{c}^{*} \bar{D}$. Moreover, their respective hidden-color channels increase their attraction a little. No bound state can be obtained in the 
Table 3 The results for

$I J^{P}=0 \frac{1}{2}^{-}$. cc1: mixing of color singlet-singlet channels, cc2: mixing of all channels (unit: MeV)

\begin{tabular}{|c|c|c|c|c|c|c|c|c|c|}
\hline Index & $\psi^{f_{i}}$ & $\psi^{\sigma_{j}}$ & $\psi^{c_{k}}$ & Physical channel & $\mathrm{E}$ & $E_{\text {th }}^{\text {theo }}$ & $E_{B}$ & $E_{t h}^{e x p}$ & $E^{\prime}$ \\
\hline 1 & $i=1$ & $j=2$ & $k=1$ & $\Lambda \eta_{c}$ & 4098 & 4098 & 0 & 4100 & 4100 \\
\hline 2 & $i=1$ & $j=1,2$ & $k=1,2,3$ & & 4098 & & & & \\
\hline 3 & $i=1$ & $j=4$ & $k=1$ & $\Lambda J / \psi$ & 4296 & 4296 & 0 & 4213 & 4213 \\
\hline 4 & $i=1$ & $j=3,4$ & $k=1,2,3$ & & 4296 & & & & \\
\hline 5 & $i=2$ & $j=2$ & $k=1$ & $\Lambda_{c} \bar{D}_{s}$ & 4148 & 4148 & 0 & 4250 & 4250 \\
\hline 6 & $i=2$ & $j=1,2$ & $k=1,2,3$ & & 4148 & & & & \\
\hline 7 & $i=2$ & $j=4$ & $k=1$ & $\Lambda_{c}{\overline{D_{s}}}^{*}$ & 4340 & 4340 & 0 & 4398 & 4398 \\
\hline 8 & $i=2$ & $j=3,4$ & $k=1,2,3$ & & 4340 & & & & \\
\hline 9 & $i=3$ & $j=2$ & $k=1$ & $\Xi_{c} \bar{D}$ & 4313 & 4318 & -5 & 4335 & 4330 \\
\hline 10 & $i=3$ & $j=1,2$ & $k=1,2,3$ & & 4312 & & -6 & & 4329 \\
\hline 11 & $i=3$ & $j=4$ & $k=1$ & $\Xi_{c} \bar{D}^{*}$ & 4480 & 4483 & -3 & 4478 & 4475 \\
\hline 12 & $i=3$ & $j=3,4$ & $k=1,2,3$ & & 4478 & & -5 & & 4473 \\
\hline 13 & $i=4$ & $j=1$ & $k=1$ & $\Xi_{c}^{\prime} \bar{D}$ & 4436 & 4439 & -3 & 4443 & 4440 \\
\hline 14 & $i=4$ & $j=1,2$ & $k=1,2,3$ & & 4435 & & -4 & & 4439 \\
\hline 15 & $i=4$ & $j=3$ & $k=1$ & $\Xi_{c}^{\prime} \bar{D}^{*}$ & 4604 & 4604 & 0 & 4586 & 4586 \\
\hline 16 & $i=4$ & $j=3,4$ & $k=1,2,3$ & & 4604 & & & & \\
\hline 17 & $i=4$ & $j=5$ & $k=1$ & $\Xi_{c}^{*} \bar{D}^{*}$ & 4676 & 4676 & 0 & 4652 & 4652 \\
\hline 18 & $i=4$ & $j=5$ & $k=1,2,3$ & & 4676 & & & & \\
\hline $\mathrm{cc} 1$ & & & & & 4098 & & 0 & & \\
\hline $\operatorname{cc} 2$ & & & & & 4098 & & & & \\
\hline
\end{tabular}

Table 4 The results for $I J^{P}=0 \frac{3}{2}^{-}$. cc1: mixing of color singlet-singlet channels, cc2: mixing of all channels (unit: MeV)

\begin{tabular}{|c|c|c|c|c|c|c|c|c|c|}
\hline Index & $\psi^{f_{i}}$ & $\psi^{\sigma_{j}}$ & $\psi^{c_{k}}$ & Physical channel & $\mathrm{E}$ & $E_{t h}^{\text {Theo }}$ & $E_{B}$ & $E_{t h}^{\operatorname{Exp}}$ & $E^{\prime}$ \\
\hline 1 & $i=1$ & $j=7$ & $k=1$ & $\Lambda J / \psi$ & 4296 & 4296 & 0 & 4213 & 4213 \\
\hline 2 & $i=1$ & $j=6,7$ & $k=1,2,3$ & & 4296 & & & & \\
\hline 3 & $i=2$ & $j=7$ & $k=1$ & $\Lambda_{c} \bar{D}_{s}{ }^{*}$ & 4340 & 4340 & 0 & 4398 & 4398 \\
\hline 4 & $i=2$ & $j=6,7$ & $k=1,2,3$ & & 4340 & & & & \\
\hline 5 & $i=3$ & $j=7$ & $k=1$ & $\Xi_{c} \bar{D}^{*}$ & 4481 & 4483 & -2 & 4478 & 4476 \\
\hline 6 & $i=3$ & $j=6,7$ & $k=1,2,3$ & & 4480 & & -3 & & 4475 \\
\hline 7 & $i=4$ & $j=7$ & $k=1$ & $\Xi_{c}^{\prime} \bar{D}^{*}$ & 4600 & 4604 & -4 & 4586 & 4582 \\
\hline 8 & $i=4$ & $j=6,7$ & $k=1,2,3$ & & 4599 & & -5 & & 4581 \\
\hline 9 & $i=4$ & $j=8$ & $k=1$ & $\Xi_{c}^{*} \bar{D}$ & 4510 & 4511 & -1 & 4509 & 4508 \\
\hline 10 & $i=4$ & $j=8$ & $k=1,2,3$ & & 4510 & & -1 & & 4508 \\
\hline 11 & $i=4$ & $j=9$ & $k=1$ & $\Xi_{c}^{*} \bar{D}^{*}$ & 4676 & 4676 & 0 & 4652 & 4652 \\
\hline 12 & $i=4$ & $j=9$ & $k=1,2,3$ & & 4676 & & & & \\
\hline $\mathrm{cc} 1$ & & & & & 4296 & & 0 & & \\
\hline $\operatorname{cc} 2$ & & & & & 4296 & & & & \\
\hline
\end{tabular}

Table 5 The results for $I J^{P}=0 \frac{5}{2}^{-}$

\begin{tabular}{llllllllll}
\hline Index & $\psi^{f_{i}}$ & $\psi^{\sigma_{j}}$ & $\psi^{c_{k}}$ & Physical channel & $\mathrm{E}$ & $E_{\text {th }}^{\text {Theo }}$ & $E_{B}$ & $E_{t h}^{E x p}$ & $E^{\prime}$ \\
\hline 1 & $i=4$ & $j=10$ & $k=1$ & $\Xi_{c}^{*} \bar{D}^{*}$ & 4672 & 4676 & -4 & 4652 & 4648 \\
2 & $i=4$ & $j=10$ & $k=1,2,3$ & & 4671 & & -5 & & 4647 \\
\hline
\end{tabular}


color singlet-singlet and full channel coupling calculations. Resonances are still possible.

(c) For $I J^{P}=0 \frac{5}{2}^{-}$system in Table 5, there is only one color singlet-singlet channel $\left(\Xi_{c}^{*} \bar{D}^{*}\right)$ and there exist a bound state with binding energy $\sim 5 \mathrm{MeV}$. Generally attractions exist between two vector hadrons, and the energy of the system is higher than the sum of two pseudoscalar mesons, resonances are always expected here because of the high angular momentum of the state, which make the coupling between vector-vector mesons channel and pseudoscalar-pseudoscalar mesons channel via the tensor interaction. Thus, $\Xi_{c}^{*} \bar{D}^{*}$ can be a good candidate for pentaquark and more experimental data are needed in the future.

We compared our single-channel calculation results with that of other work and found that our results are qualitatively consistent with that of other work although different models and calculation methods are used, but the binding energies of states obtained in our work are generally smaller. For example, the binding energy of $\left[\Xi_{c} \bar{D}\right]_{1 / 2}$ is $6 \mathrm{MeV}$, whereas it is 13 $\mathrm{MeV}$ in Ref. [28], $58 \mathrm{MeV}$ in Ref. [42], and 0.9-27 MeV in Ref. [43]. Then it is naturally to expect that some states with small binding energy in other work will become unbound states in our calculation. $\Xi_{c}^{*} \bar{D}^{*}$ has binding energy $1 \mathrm{MeV}$ in Ref. [28], it become an unbound state in our calculation. The argument for the unbound of the state in our work is that the $\sigma$-exchange potential plays a role only once, between $u, d$ quarks, compared with $P_{c}$ calculation.

To illustrate the mechanism of forming a bound state, the contributions from each term in Hamiltonian are listed in Table 6 , The values in the table are the differences between the contribution of each term in the five-quark system and the sum of its contribution in two individual hadrons. From the table, we can see that the OGE and $\sigma$-exchange potentials cancel the contribution of kinetic energy and make the state bound. $\eta$-meson exchange is always attractive and $\pi$-meson exchange can be attractive or repulsive. For the unbound states, $\left[\Xi_{c}^{\prime} \bar{D}^{*}\right]_{\frac{1}{2}},\left[\Xi_{c}^{*} \bar{D}^{*}\right]_{\frac{1}{2}}$ and $\left[\Xi_{c}^{*} \bar{D}^{*}\right]_{\frac{3}{2}}$, the contributions from OGE and $\sigma$-exchange are not strong to bind the two hadrons together, the separation between two hadrons increases, which in turn make the contributions of each term smaller.

To check whether the resonances with quantum numbers $I J^{P}=0 \frac{1}{2}^{-}, 0 \frac{3}{2}^{-}$can survive after coupling to the open channels, a stability method to identify genuine resonance states, real-scaling method [38-40], is employed. In this method, the Gaussian size parameter $r_{n}$ for the basis functions between baryon and meson clusters for the colorsinglet channels is scaled by multiplying a factor $\alpha: r_{n} \longrightarrow$ $\alpha r_{n}$. As a result, a genuine resonance will act as an avoidcrossing structure (see Fig. 1) with the increasing of $\alpha$, while
Table 6 Contributions of each term in Hamiltonian to the binding energy for possible $\Xi_{c}^{(/ / *)} \bar{D}^{(*)}$ systems (unit: Mev)

\begin{tabular}{llllllll}
\hline & $\mathrm{K}$ & Conf. & OGE & $\pi$ & $\eta$ & $\sigma$ & $B . E$ \\
\hline$\left[\Xi_{c} \bar{D}\right]_{\frac{1}{2}}$ & 15.8 & -0.3 & -17.7 & 4.4 & -0.9 & -5.7 & -5 \\
{$\left[\Xi_{c} \bar{D}^{*}\right]_{\frac{1}{2}}$} & 9.1 & 0.4 & -10.2 & 2.9 & -0.6 & -4.0 & -3 \\
{$\left[\Xi_{c} \bar{D}^{*}\right]_{\frac{3}{2}}$} & 7.8 & 0.6 & -8.7 & 2.7 & -0.5 & -3.7 & -2 \\
{$\left[\Xi_{c}^{\prime} \bar{D}\right]_{\frac{1}{2}}$} & 7.4 & -0.1 & -8.4 & 2.7 & -0.2 & -3.7 & -5 \\
{$\left[\Xi_{c}^{\prime} \bar{D}^{*}\right]_{\frac{1}{2}}$} & 2.6 & -0.3 & -0.5 & -0.4 & -0.03 & -0.4 & 0 \\
{$\left[\Xi_{c}^{*} \bar{D}^{*}\right]_{\frac{1}{2}}$} & 2.3 & -0.3 & -0.3 & -0.3 & -0.02 & -0.3 & 0 \\
{$\left[\Xi_{c}^{\prime} \bar{D}^{*}\right]_{\frac{3}{2}}$} & 1.7 & 1.6 & -8.8 & 5.8 & -0.22 & -3.9 & -4 \\
{$\left[\Xi_{c}^{*} \bar{D}\right]_{\frac{3}{2}}$} & 5.5 & 0.2 & -6.2 & 2.2 & -0.03 & -3.1 & -1 \\
{$\left[\Xi_{c}^{*} \bar{D}^{*}\right]_{\frac{3}{2}}$} & 2.7 & -0.1 & -1.1 & -0.1 & -0.1 & -0.8 & 0 \\
{$\left[\Xi_{c}^{*} \bar{D}^{*}\right]_{\frac{5}{2}}$} & 0.6 & 2.1 & -9.5 & 7.3 & -0.2 & -4.0 & -4 \\
\hline
\end{tabular}

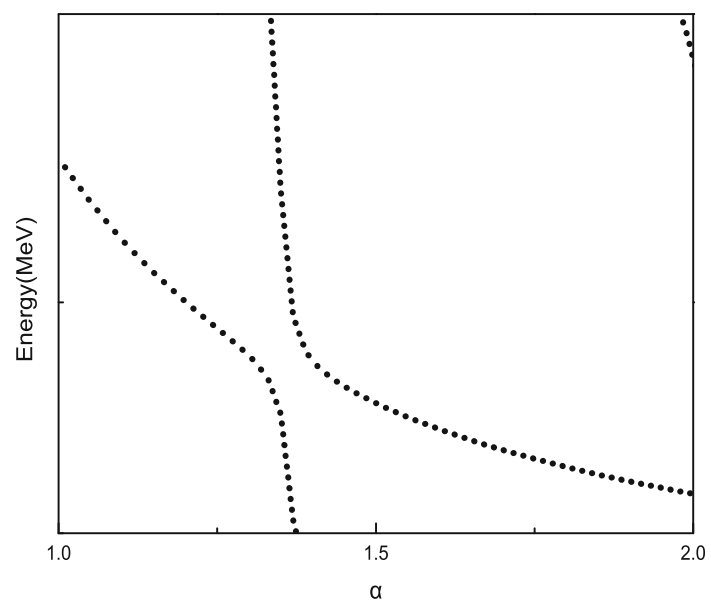

Fig. 1 The shape of the resonance in real-scaling method

other continuum states will fall off towards its threshold. If the avoid-crossing structure is repeated periodically as $\alpha$ increase, then the avoid-crossing structure is a genuine resonance [41].

The results are shown in Figs. 2, 3 and 4. In these figures, the thresholds of all physical channels appear as horizontal lines and are marked with lines (red lines), tagged with their contents. And for genuine resonances, which appear as avoid-crossing structure and are marked with blue lines. The continuum states fall off towards their respective threshold states (red horizontal lines).

For $I J^{P}=0 \frac{1}{2}^{-}$system, we get two resonances whose energy are $4301 \mathrm{MeV}$ (main component is $\Xi_{c} \bar{D}$ ) and 4461 $\mathrm{MeV}$ (main component is $\Xi_{c} \bar{D}^{*}$ ). Especially for $P_{c s}(4461)$, which is very close to $4459 \mathrm{MeV}$, it is a good candidate for $P_{c s}(4459)$ reported by $\mathrm{LHCb}$ Collaboration. In $I J^{P}=0 \frac{3}{2}^{-}$ system, there are three resonances, $P_{c s}$ (4443) (main component is $\left.\Xi_{c} \bar{D}^{*}\right), P_{c s}(4500)$ (main component is $\Xi_{c}^{*} \bar{D}$ ) and 

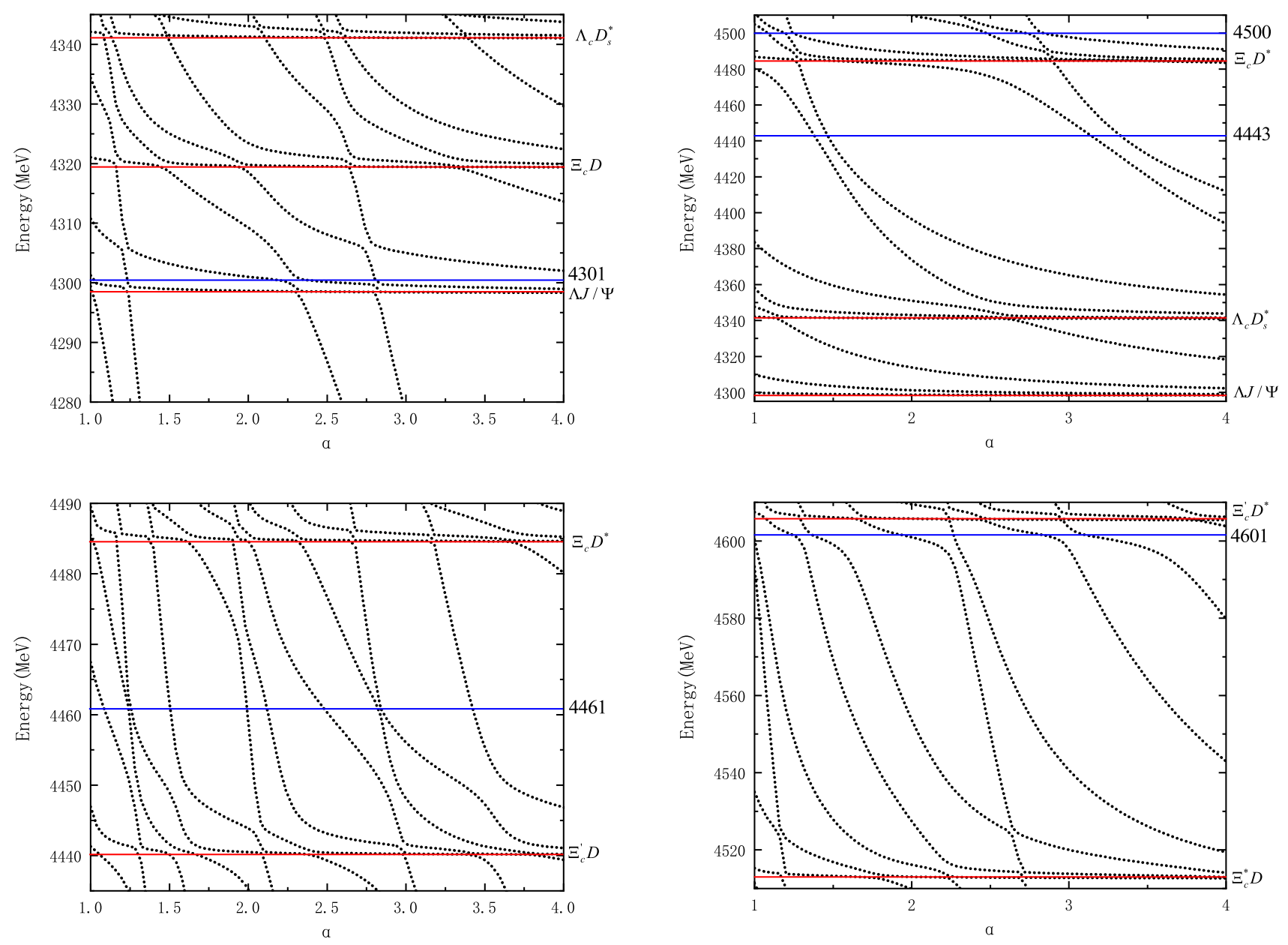

Fig. 2 Energy spectrum of $0 \frac{1}{2}^{-}$system

$P_{c S}$ (4601) (main component is $\Xi_{c}^{\prime} \bar{D}^{*}$ ). Finally, in $I J^{P}=$ $0 \frac{5}{2}^{-}$system, there is only one bound state marked horizontally under its threshold $\left(P_{c s}(4671)\right)$, it will turn to a narrow resonance state after coupling to $\Xi_{c} \bar{D}$ via tensor interaction.

It is worth mentioning that in case we use real-scaling method to identify resonances, we will also calculate the composition of the possible resonances to find the mechanism of the formation the resonances. The main component of a genuine resonance should be bound state channels in the single channel or coupling channel (the main component channel and other channels with energies higher than the main component channel) calculations. In Figs. 2, 3, in addition to the resonances found, there are other avoid-crossing structures such as $4306 \mathrm{MeV}$ in $I J^{P}=0 \frac{1}{2}^{-}$system and $4525 \mathrm{MeV}$ in $I J^{P}=0 \frac{3}{2}^{-}$system. However, their components are open channels, which means the avoid-crossing structures are formed due to the difference in the decay slope of different open channels.

For resonances, the partial widths of two mesons strong decay can be extracted from theses figures. The decay width

Fig. 3 Energy spectrum of $0 \frac{3}{2}^{-}$system

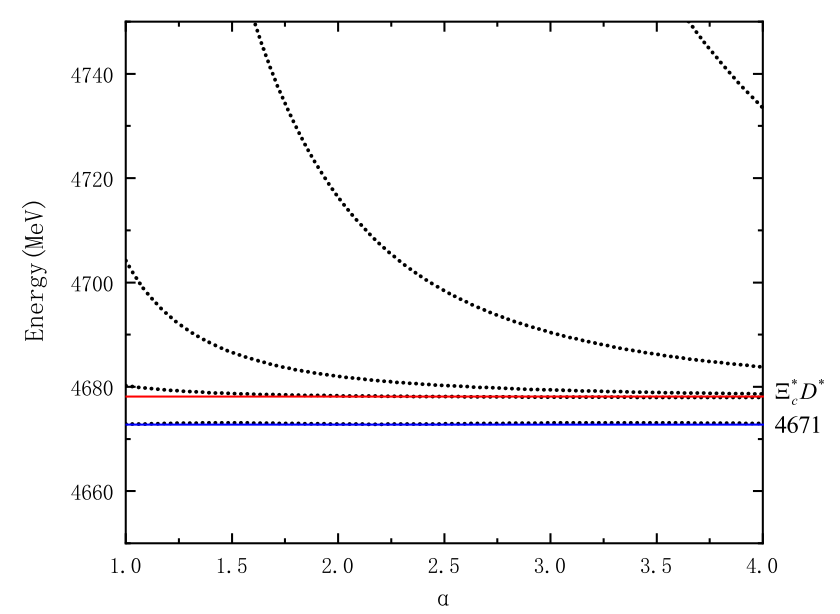

Fig. 4 Energy spectrum of $0 \frac{5}{2}^{-}$system

of resonance to possible open channels of two mesons is obtained by the following formula

$$
\Gamma=4 V(\alpha) \frac{\sqrt{\left(k_{r} \times k_{c}\right)}}{\left|k_{r}-k_{c}\right|},
$$




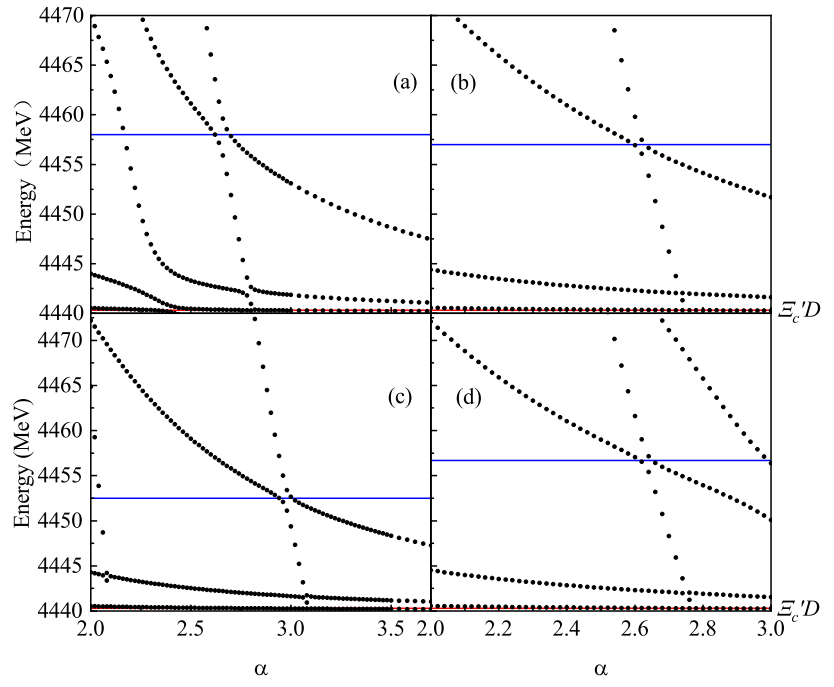

Fig. 5 Energy spectrum of $P_{c s}$ (4461) in four channels coupling, three bound state channels: $\Xi_{c} \bar{D}, \Xi_{c} \bar{D}^{*}$ and $\Xi_{c}^{\prime} \bar{D}$, and one open channel for $0 \frac{1}{2}^{-}$system. The open channel is (a) $\Lambda \eta_{c}$, (b) $\Lambda J / \psi$, (c) $\Lambda_{c} \bar{D}_{s}$, and (d) $\Lambda_{c} \bar{D}_{s}^{*}$

Table 7 The decay width of $P_{c s}$ states in $0 \frac{1}{2}^{-}$system (unit: MeV)

\begin{tabular}{lll}
\hline Mode & $P_{c s}(4301)$ & $P_{c s}(4461)$ \\
\hline Total width & 4.0 & 8.9 \\
$\Lambda \eta_{c}$ & 2.5 & 3.7 \\
$\Lambda J / \psi$ & 1.5 & 1.2 \\
$\Lambda_{c} \bar{D}_{s}$ & $?$ & 1.5 \\
$\Lambda_{c} \bar{D}_{s}^{*}$ & - & 2.5 \\
Main comp. & $\Xi_{c} \bar{D}$ & $\Xi_{c} \bar{D}^{*}$ \\
$E^{\prime}$ & 4318 & 4458 \\
\hline
\end{tabular}

where $V(\alpha)$ is the minimum energy difference, while $k_{c}$ and $k_{r}$ stand for the slopes of scattering state and resonance state respectively. More details can be found in Ref. [38].

To extract the partial decay to specific channel, more calculations are needed. For example, to obtain the partial decay width of resonance $P_{c s}(4461)$ in $I J^{P}=0 \frac{1}{2}^{-}$system, the channel coupling calculations with three bound state channels, $\Xi_{c} \bar{D}, \Xi_{c} \bar{D}^{*}$ and $\Xi_{c}^{\prime} \bar{D}$ and one of four open channels: $\Lambda \eta_{c}, \Lambda J / \psi, \Lambda_{c} \bar{D}_{s}$, and $\Lambda_{c} \bar{D}_{s}{ }^{*}$ are performed, the results are shown in Fig. 5. From Fig. 5a, one can extract the partial decay width of $P_{c S}(4461)$ to $\Lambda \eta_{c}$ and so on. In different channel coupling calculations, the obtained resonance energies are slightly different, from 4453 to $4458 \mathrm{MeV}$. It is due to the finite model space used, the avoid-crossing structures appear at different scaling factors. Finally, all possible resonances and their respective partial decay widths and total decay width to two ground hadrons are shown in the Tables 6 and 7. The main component of each resonance and corrected energy are also given in the tables.
Table 8 The decay width of $P_{c s}$ states in $0 \frac{3}{2}^{-}$system (unit: $\mathrm{MeV}$ )

\begin{tabular}{llll}
\hline Mode & $P_{c s}(4443)$ & $P_{c s}(4500)$ & $P_{c s}(4601)$ \\
\hline Total width & 24.4 & 7.3 & 5.9 \\
$\Lambda J / \psi$ & 19.6 & 1.7 & 3.3 \\
$\Lambda_{c} \bar{D}_{s}^{*}$ & 4.8 & 5.6 & 2.6 \\
Main comp. & $\Xi_{c} \bar{D}^{*}$ & $\Xi_{c}^{*} \bar{D}$ & $\Xi_{c}^{\prime} \bar{D}^{*}$ \\
$E^{\prime}$ & 4444 & 4497 & 4585 \\
\hline
\end{tabular}

From Tables 7 and 8 , we can see that, in $0 \frac{1}{2}^{-}$system, the total decay width of $P_{c s}(4301)$ is $4.0 \mathrm{MeV}$ and we found its decay width to channel $\Lambda \eta_{c}$ is very narrow. However, we found no typical avoid-crossing structures when we calculated its decay width to channel $\Lambda_{c} \bar{D}_{s}$, which is a problem that needs further study. For $P_{c s}(4461)$, the decay width is 8.9 $\mathrm{MeV}$ and $\Lambda \eta_{c}$ is the main decay channel. In $0 \frac{3}{2}^{-}$system, the decay width of two resonances $P_{c s}(4500)$ and $P_{c s}(4601)$ are $7.3 \mathrm{MeV}$ and $5.9 \mathrm{MeV}$, the main decay channel of $P_{c s}(4500)$ is $\Lambda_{c} \bar{D}_{s}{ }^{*}$, and $P_{c s}(4601)$ has comparable decay widths to $\Lambda_{c} \bar{D}_{s}{ }^{*}$ and $\Lambda J / \psi$. For $P_{c s}(4443)$, the decay width is 24.4 $\mathrm{MeV}$ and its main decay channel is $\Lambda J / \psi$. For $0 \frac{5}{2}^{-}$system, there is only resonance $P_{c s}(4671)$, which is $5 \mathrm{MeV}$ under its threshold and the decay width is estimated to be $5-15 \mathrm{MeV}$ due to decay widths of its constituents, $\Xi_{c}^{*}\left(\Gamma_{\Xi_{c}^{*} \longrightarrow \Xi_{c} \pi} \sim 2.35\right.$ $\mathrm{MeV})$ and $\bar{D}^{*}\left(\Gamma_{D^{*} \longrightarrow D \pi} \sim 2 \mathrm{MeV}\right)$ and the decay width to $\Xi_{c} \bar{D}, 1 \sim 10 \mathrm{MeV}$, via tensor interaction. Considering the deviation of quark model calculations and the uncertainty of experimental results, $P_{c s}(4461)$ in $0 \frac{1}{2}^{-}$system can be identified as $P_{c s}(4459)$ reported by LHCb Collaboration.

\section{Summary}

In this paper, the hidden-charm pentaquark systems with single strangeness is investigated in chiral quark model. The calculation shows that there are several states in systems $0 \frac{1}{2}^{-}, 0 \frac{3}{2}^{-}$and $0 \frac{5}{2}^{-}$, which means good resonances can be formed. Real-scaling method are used to check the genuine resonances and study their decay width. In $0 \frac{1}{2}^{-}$system, two resonances $P_{C S}(4301)$ and $P_{C S}(4461)$ have been found. In particular, $P_{c s}(4461)$, whose main component is $\Xi_{c} \bar{D}^{*}$, is regarded as a good candidate for $P_{c s}(4459)$ recently reported by $\mathrm{LHCb}$ Collaboration. Other possible pentaquarks are also predicted. Three states, $P_{c s}(4443), P_{c S}(4500)$ and $P_{c S}(4601)$ are found in system $0 \frac{3}{2}^{-}$. It is worth mentioning that there are $P_{c S}(4461)$ and $P_{c S}(4443)$ in our calculations, which can form a two-peak structure composed of $\Xi_{c} \bar{D}^{*}$ states with quantum number of $0 \frac{1}{2}^{-}$and $0 \frac{3}{2}^{-}$, similar to $P_{c}(4440)$ and $P_{c}(4457)$. Finally, only one molecule state $\Xi_{c}^{*} \bar{D}^{*}$ exists in system $0 \frac{5}{2}^{-}$and it is a good candidate for heavy pentaquark 
with high spin with its decay width is in range of $5 \mathrm{MeV}$ to $15 \mathrm{MeV}$.

From the results, one can see that the behaviors of $P_{c s}$ systems are similar to that of $P_{c}$ systems. Due to the success of chiral quark model on $P_{c}$ states [32], it is expected that the possible resonances proposed above can be searched in future experiments.

Acknowledgements The work is supported partly by the National Natural Science Foundation of China under Grant Nos. 11775118, and 11535005

Data Availability Statement This manuscript has no associated data or the data will not be deposited. [Authors' comment: The data have been illustrated in the figures and tables, so they are not necessary to be deposited. Data may be made available upon request.]

Open Access This article is licensed under a Creative Commons Attribution 4.0 International License, which permits use, sharing, adaptation, distribution and reproduction in any medium or format, as long as you give appropriate credit to the original author(s) and the source, provide a link to the Creative Commons licence, and indicate if changes were made. The images or other third party material in this article are included in the article's Creative Commons licence, unless indicated otherwise in a credit line to the material. If material is not included in the article's Creative Commons licence and your intended use is not permitted by statutory regulation or exceeds the permitted use, you will need to obtain permission directly from the copyright holder. To view a copy of this licence, visit http://creativecomm ons.org/licenses/by/4.0/.

Funded by SCOAP ${ }^{3}$.

\section{References}

1. D. Diakonov, V. Petrov, M. Polyakov, Z. Phys. A 359, 305 (1997)

2. T. Nakano et al., [LEPS Collaboration], Phys. Rev. Lett. 91, 012002 (2003)

3. R. Aaij et al., [LHCb Collaboration], Phys. Rev. Lett. 115, 072001 (2015)

4. R. Aaij et al., [LHCb Collaboration], Phys. Rev. Lett. 122, 222001 (2019)

5. R. Chen, X. Liu, S.L. Zhu, Nucl. Phys. A 954, 406-421 (2016)

6. H.X. Chen, E.L. Cui, W. Chen, X. Liu, T.G. Steele, S.L. Zhu, Eur. Phys. J. C 76, 572 (2016)

7. Z.G. Wang, Eur. Phys. J. C 76, 70 (2016)

8. Q.F. Lu, Y.B. Dong, Phys. Rev. D 93, 074020 (2016)

9. J. He, Eur. Phys. J. C 79, 393 (2019)

10. M.Z. Liu, Y.W. Pan, F.Z. Peng, M.S. Sánchez, L.S. Geng, A. Hosaka, M.P. Valderrama, Phys. Rev. Lett. 122, 242001 (2019)
11. C.J. Xiao, Y. Huang, Y.B. Dong, L.S. Geng, D.Y. Chen, Phys. Rev. D 100, 014022 (2019)

12. L. Maiani, A.D. Polosa, V. Riquer, Phys. Lett. B 749, 289 (2015)

13. C.R. Deng, J.L. Ping, H.X. Huang, F. Wang, Phys. Rev. D 95, $014031(2017)$

14. C.-J. Xiao, Y. Huang, Y.-B. Dong, L.S. Geng, D.-Y. Chen, Phys. Rev. D 100, 014022 (2019)

15. R. Aaij et al. [LHCb Collaboration], arXiv:2012.10380 [hep-ex]

16. H.X. Chen, W. Chen, X. Liu, X.H. Liu, arXiv:2011.01079 [hep-ph]

17. Z.G. Wang, arXiv:2011.05102 [hep-ph]

18. C.W. Xiao, J.J. Wu, B.S. Zou, Phys. Rev. D 103, 054016 (2021)

19. F.Z. Peng, M.J. Yan, M.S. Sánchez, M.P. Valderrama, arXiv:2011.01915 [hep-ph]

20. Rui Chen, Eur. Phys. J. C 81, 122 (2021)

21. W.Y. Liu, W. Hao, G.Y. Wang, Y.Y. Wang, E. Wang, Phys. Rev. D 103, 034019 (2021)

22. R. Chen, Phys. Rev. D 103, 054007 (2021)

23. J.-T. Zhu, L.-Q. Song, J. He, Phys. Rev. D 103, 074007 (2021)

24. K. Azizi, Y. Sarac, H. Sundu, Phys. Rev. D 103, 094033 (2021)

25. A. Feijoo, V.K. Magas, A. Ramos, E. Oset, Eur. Phys. J. C 76, 446 (2016)

26. J.X. Lu, E. Wang, J.J. Xie, L.S. Geng, E. Oset, Phys. Rev. D 93, 094009 (2016)

27. H.X. Chen, L.S. Geng, W.H. Liang, E. Oset, E. Wang, J.J. Xie, Phys. Rev. C 93, 065203 (2016)

28. B. Wang, L. Meng, S.L. Zhu, Phys. Rev. D 101, 034018 (2020)

29. J.J. Wu, R. Molina, E. Oset, B.S. Zou, Phys. Rev. Lett. 105, 232001 (2010)

30. J.J. Wu, R. Molina, E. Oset, B.S. Zou, Phys. Rev. C 84, 015202 (2011)

31. Z.C. Yang, Z.F. Sun, J. He, X. Liu, S.L. Zhu, Chin. Phys. C 36, 6 (2012)

32. H.X. Huang, J.L. Ping, Phys. Rev. D 99, 014010 (2019)

33. M. Gell-Mann, Phys. Lett. 8, 214 (1964)

34. G. Zweig, CERN-TH-412 (2020)

35. E. Hiyama, Y. Kino, M. Kamimura, Prog. Part. Nucl. Phys. 51, 223 (2003)

36. A. Valcarce, H. Garcilazo, F. Fernandez, P. Gonzalez, Rep. Prog. Phys. 68, 965 (2005)

37. J. Vijande, F. Fernandez, A. Valcarce, J. Phys. G 31, 481 (2005)

38. J. Simons, J. Chem. Phys. 75, 2465 (1981)

39. E. Hiyama, A. Hosaka, M. Oka, J.M. Richard, Phys. Rev. C 98, 045208 (2018)

40. Q. Meng, E. Hiyama, K.U. Can, P. Gubler, M. Oka, A. Hosaka, H. Zong, Phys. Lett. B 798, 135028 (2019)

41. Y. Tan, J.L. Ping, Chin. Phys. C 45, 093104 (2021)

42. C.W. Xiao, J. Nieves, E. Oset, Phys. Lett. B 799, 135051 (2019)

43. M.Z. Liu, Y.W. Pan, L.S. Geng, Phys. Rev. D 103, 034003 (2021) 